\title{
Epidemiometric modelling in leprosy based on Indian data
}

\author{
M F LECHAT
}

\section{Introduction}

Today, I suppose, any moderately bright child who has had a minimal exposure to computers could programme an epidemiometric model on a rainy Sunday afternoon using existing softwares and a technologically basic machine, but the situation was quite different in the early 1970s when the leprosy epidemiometric model was first designed.

The problem addressed was clearly circumscribed. After 20 years of large scale, mass control campaigns based on dapsone monotherapy, leprosy had not been eradicated, and even worse, it was not known whether the disease was or was not on the decrease. International funding agencies such as UNICEF, nongovernmental organizations, as well as governments, all were getting tired of emphatic promises and overdue delays. There were talks of a vaccine which could revolutionize the control strategy.

The concerns at that time were: (1) was it reasonable to expect a decline of the leprosy problem in the next 20 years, using current control methods? How much of a decline? How long would this take? (2) Could some improvements in the implementation of control, such as earlier detection or better compliance, speed up the decline? (3) Could some radical changes in the strategy, such as old-fashioned isolation or futuristic vaccination, modify the prediction? In what direction, and by how much?

The model aimed at predicting the trends in incidence over 20 years by using the control methods of that day. It also attempted to simulate the trends which resulted from changes in the control parameters. The indicator used was incidence, i.e. the number of new cases per year in the population.

\section{Structure}

As a first step, the development of the model required the definition of a structure and the identification of the various population subgroups (stages) as well as the permitted transitions and their directions (Figure 1). The stages were:

healthy susceptible;

latent;

multibacillary patients, nontreated, treated for less than 1 year, treated for 1 year or more, dropped from treatment, discharged;

paucibacillary patients, with the same categorization as the multibacillary patients. 


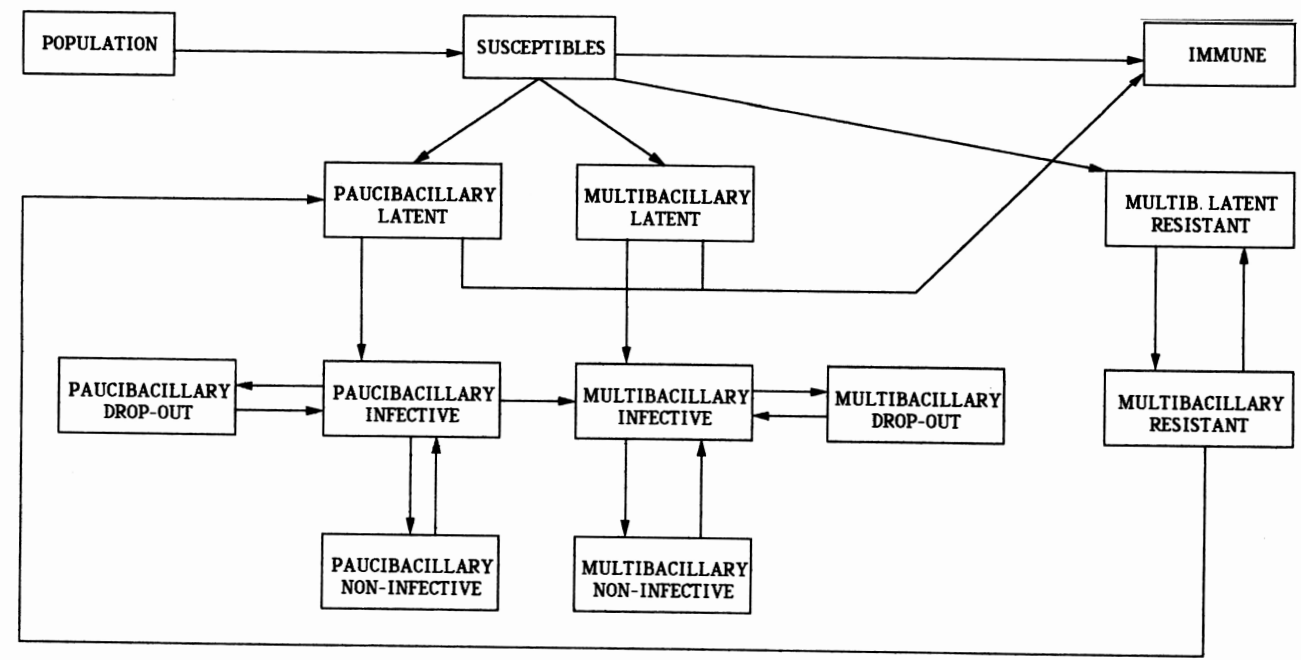

Figure 1. Model structure. Reproducted by kind permission of the Bulletin de l'Organisation mondiale de la Santé, ${ }^{2}$

For the sake of keeping it simple, the structure of the model is based on a number of epidemiological assumptions, such as no discharge without treatment, no reversal reaction, no extra-human reservoir, a constant age distribution of the population, and no migration out of or into the area.

The model is macroscopic and deterministic. It is macroscopic since it intends to describe the evolution of incidence and prevalence of the disease in the entire population, or in subpopulations, rather than in foci or at the individual level. It is deterministic because it only considers the prevalences and incidences as averages, and does not take their variability into account.

\section{Estimation of parameters}

The equation of the model was based on actual data. We were quite fortunate to have access to the files that had been assembled for nearly 16 years (1955-70) at the Leprosy Centre, Polambakkam, South India. They were allocated first by Dr F Hemerijckx, then by Dr C Vellut and their respective staffs, on 35,262 patients, representing 320,000 personyears of observation. These data had distinct characteristics.

1 They were population based, i.e. the whole population of 500 villages in a circumscribed area was regularly surveyed during the period.

2 No individuals from outside the area were included.

3 Detection was carried out in a standard way by personnel trained in the same manner over the whole period.

4 Treatment remained unchanged, based on dapsone monotherapy at a weekly dose.

It is possible that some occasional divergences from this ideal pattern occurred during the period. The consensus was that such divergences, which could not be controlled a 
posteriori, were minimal. Data validation for various types of internal inconsistencies caused the rejection of less than $0 \cdot 5 \%$.

There were 28 parameters calculated from the observed data or estimated by a statistical approach. Those parameters calculated from observations were the annual population in each stage, the transition rates, the birth and death rates, and the respective proportion of multi- and paucibacillary patients.

To represent the transition from one stage to the next, 2 types of equation were used.

Equations of the first type were based on a negative exponential probability function of staying in the initial stage. They were in the form of a negative exponential. They were used to calculate the transitions from undetected cases to detected, treated to drop-out, drop-out to retreated. The second type included conditional probabilities of transition from 1 stage to another but no special function of duration in the initial stage was used.

A negative exponential function was also used to model the delay at detection. It was set up in such a way that $75 \%$ of the new patients could be detected after 1 year from the onset of the disease. To the best of the knowledge of the field staff this estimation was reasonable.

Two major parameters could not have been directly derived from actual observations and had to be estimated by a least-square method. They were (1) the latency period between infection and onset of disease for both multibacillary and paucibacillary patients, and (2) the specific infective power of these 2 patient types. The latency period was estimated to be shorter for multibacillary than for paucibacillary patients (respectively $2 \cdot 4$ and 4.0 years). These values are in clear contradiction to the periods calculated from a few actual observations in Louisiana and among US War Veterans. Estimation of the infective capacity by considering the type of leprosy yielded more consistent estimates. The risk of a person developing leprosy through contact with a multibacillary case is higher than through contact with a paucibacillary one. This agrees with the epidemiological studies of Doull. ${ }^{1}$ However, due to the high proportion of paucibacillary patients among the diseased population, those patients constitute a nonnegligible source of infection.

\section{Simulations}

A number of simulations were carried out, either at the initial stage of the model, or later by changing some of the basic epidemiological assumptions.

Incidence depending on multibacillary or paucibacillary types of leprosy was predicted over a period of 20 years with ongoing control methods. The results indicated that with dapsone monotherapy a reduction of $50 \%$ incidence could be expected after 12 years (Figure 2). We believe this conclusion was important as it showed that control of leprosy requires perseverance, firm financial commitments, and unyielding efforts. Eradication cannot be achieved overnight.

Earlier detection, resulting in an increase to $90 \%$ from $75 \%$ of the annual input of new patients detected within 1 year of onset (assuming a negative exponential detection rate over time) does not significantly change the incidence in the long run (Figure 3). Intensifying case detection, a costly and fastidious method, had been repeatedly advocated as one of the more efficient ways to improve control. Long-term incidence is slightly more sensitive to the rate of compliance to treatment. After decreasing by $50 \%$ the 


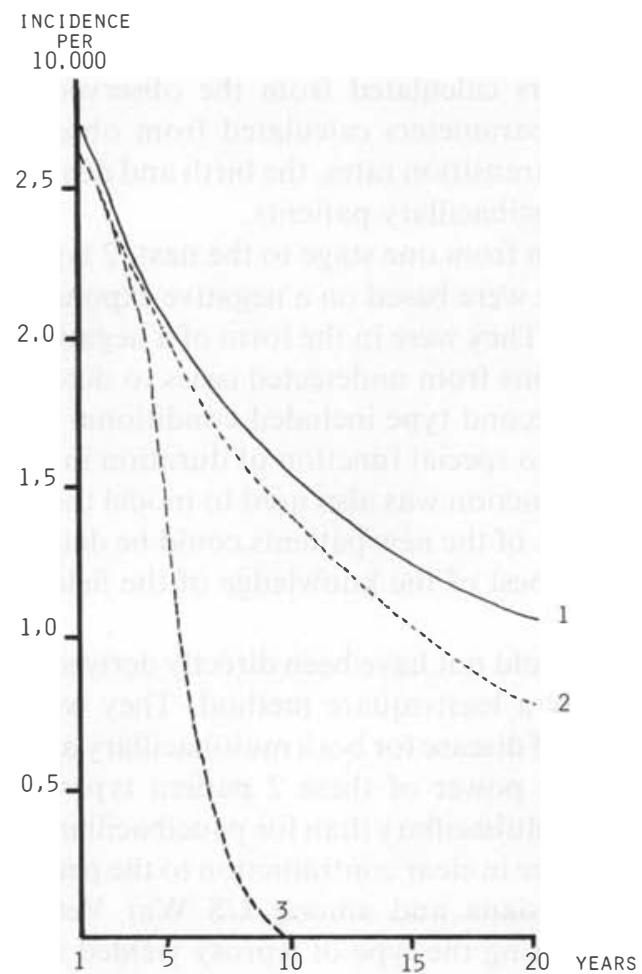

Figure 2. The incidence of 20 years:

(1) present control methods;

(2) reduction to $50 \%$ drop out; and

(3) vaccination of $100 \%$ of the population with immunoprophylactic vaccine.

annual proportion of patients who abandon treatment, incidence is reduced by $50 \%$ after 20 years (Figure 2). A cost-benefit analysis has shown that the latter method is much more advantageous than increased efforts to achieve earlier detection.

The effects of isolation were also simulated. This confirmed the largely held belief that the isolation method is futile in reducing long-term incidence (Figure 3).

The initial simulations suggested that vaccination was by far the most promising method (Figure 2). This conclusion is one of the major drawbacks of the exercise. It could have encouraged investing resources in a long-term research programme on the basis of a much too simplified model. Subsequent simulations draw a distinction between a prophylactic and a therapeutic vaccine and show that the effect of a vaccine preventing infection is delayed for several years. In the long run it is quite effective though slow for decreasing incidence (Figures 4 and 5). On the other hand, a vaccine active in preventing the appearance of leprosy in those individuals already infected has an immediate effect. It must be repeated at periodic intervals to catch those recently infected, which reduces its long-term effectiveness. In addition, the respective efficacy of using either one of these types of vaccines depends on the prevalence of infection in the population. 


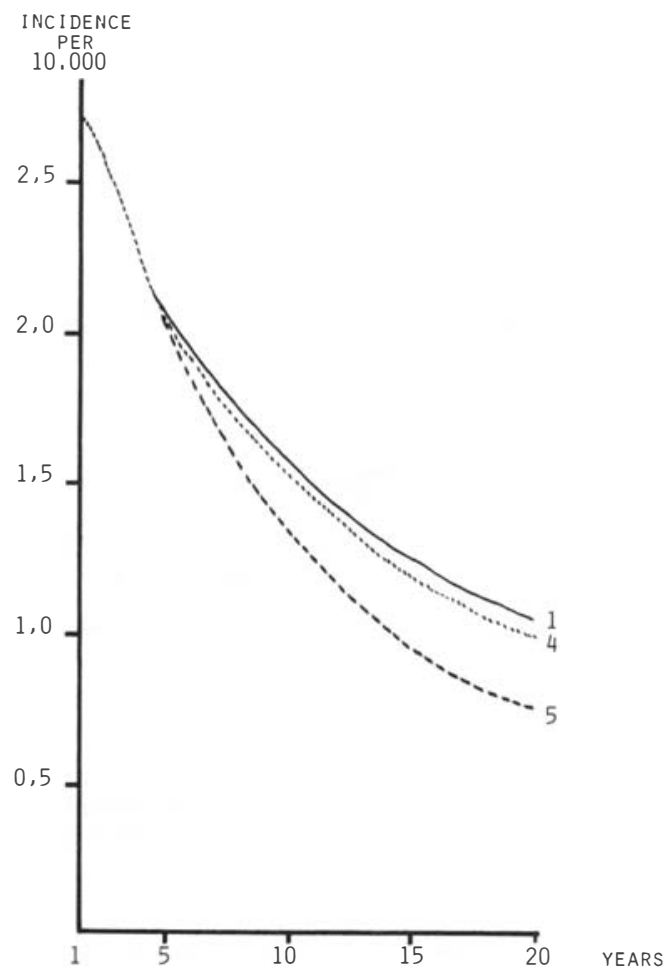

Figure 3. The incidence of 20 years:

(1) present control methods;

(4) $90 \%$ detection within one year of onset; and

(5) isolation of multibacillary cases at detection.

Simulation of various treatment regimens-dapsone monotherapy for all patients, multiple drug therapy (MDT) for all patients, MDT for multibacillary cases and dapsone therapy for the paucibacillary ones-showed that because of the ratio of multi- to paucibacillary patients which prevails in South India (about 13\% of multibacillary cases), MDT treatment for all patients, including paucibacillary, is imperative (Figure 6). Restricting MDT to the multibacillary patients is of little or no avail (for the paucibacillary patients, in spite of their low infectivity, constitute a significant source of infection because of their large numbers). This introduces the paradox that if MDT is restricted to only one type of leprosy, in this model it is most effective when only applied to paucibacillary patients.

An obvious conclusion is that it would be incorrect to restrict treatment to the paucibacillary patients even when resources are scarce. This demonstrates the paradox of individual risk vs population risk.

Simulation of various relapse rates $(10-100 \%)$ in multibacillary cases with various treatment regimens indicate that relapse with MDT is not a major problem (Figure 7). The declining trend of incidence slows down (with a $20 \%$ relapse rate) or is slightly 


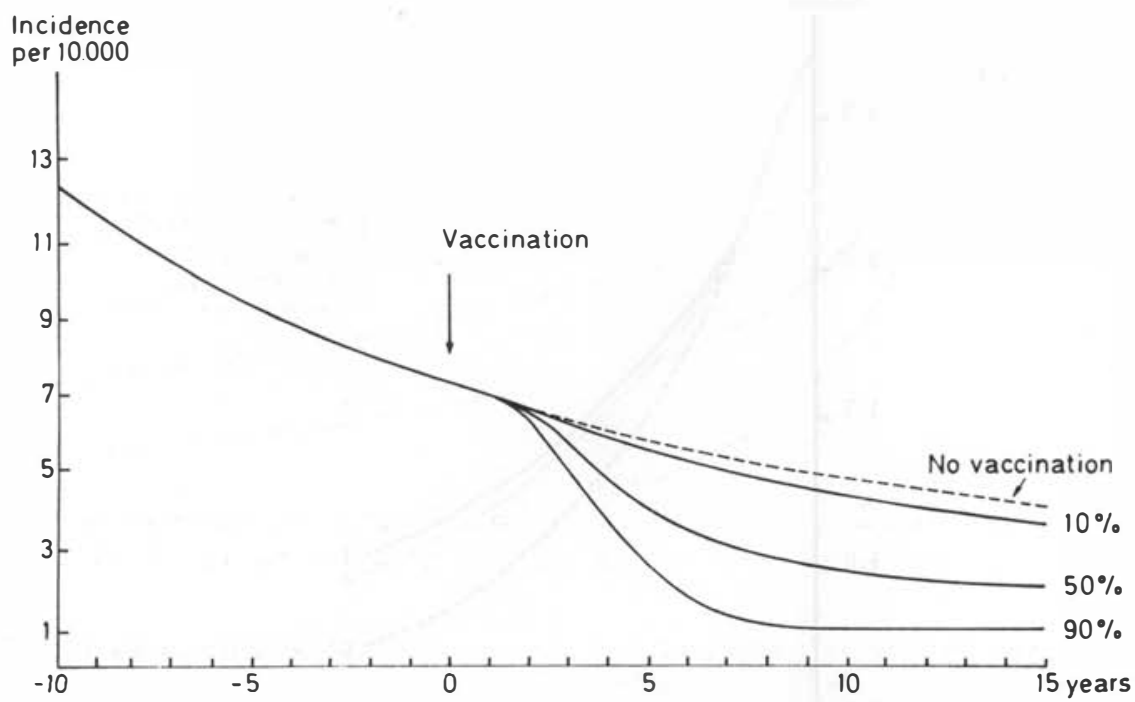

Figure 4. Prediction of incidence for both types of leprosy with an immunoprophylactic vaccine covering 10$50-90 \%$ of the population. Reproduced by kind permission of the International Journal of Le prosy. ${ }^{3}$

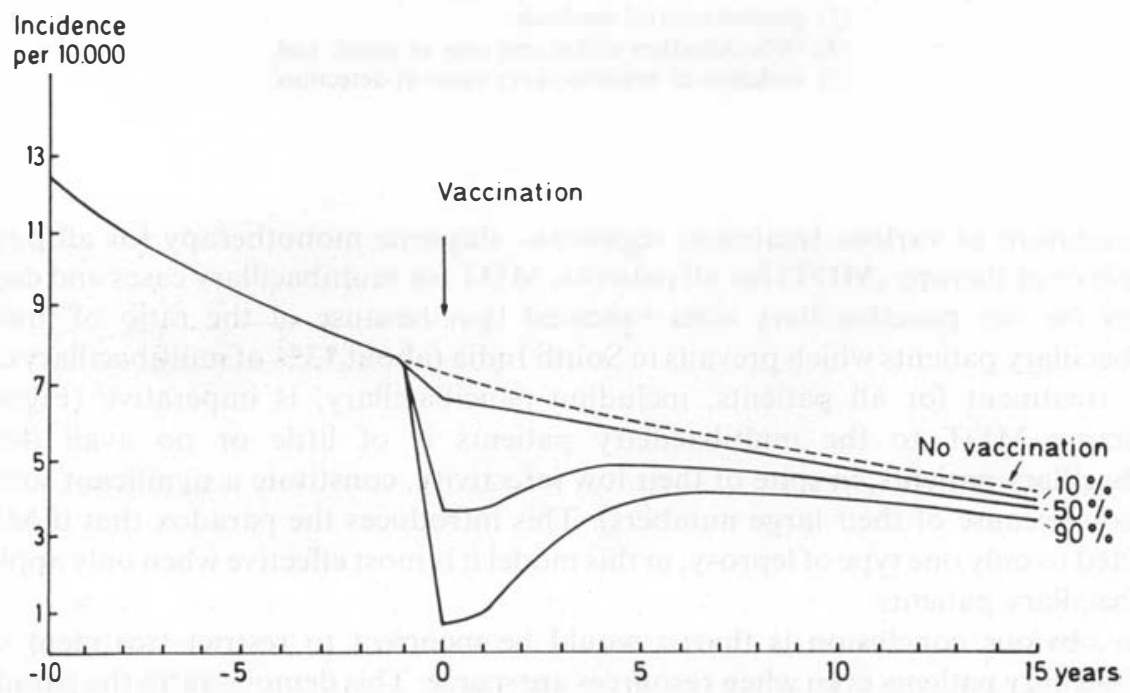

Figure 5. Prediction of incidence for both types of leprosy with an immunotherapeutic vaccine covering 10 $50-90 \%$ of the population. Reproduced by kind permission of the International Journal of Leprosy. ${ }^{3}$ 
INC. (0/00)

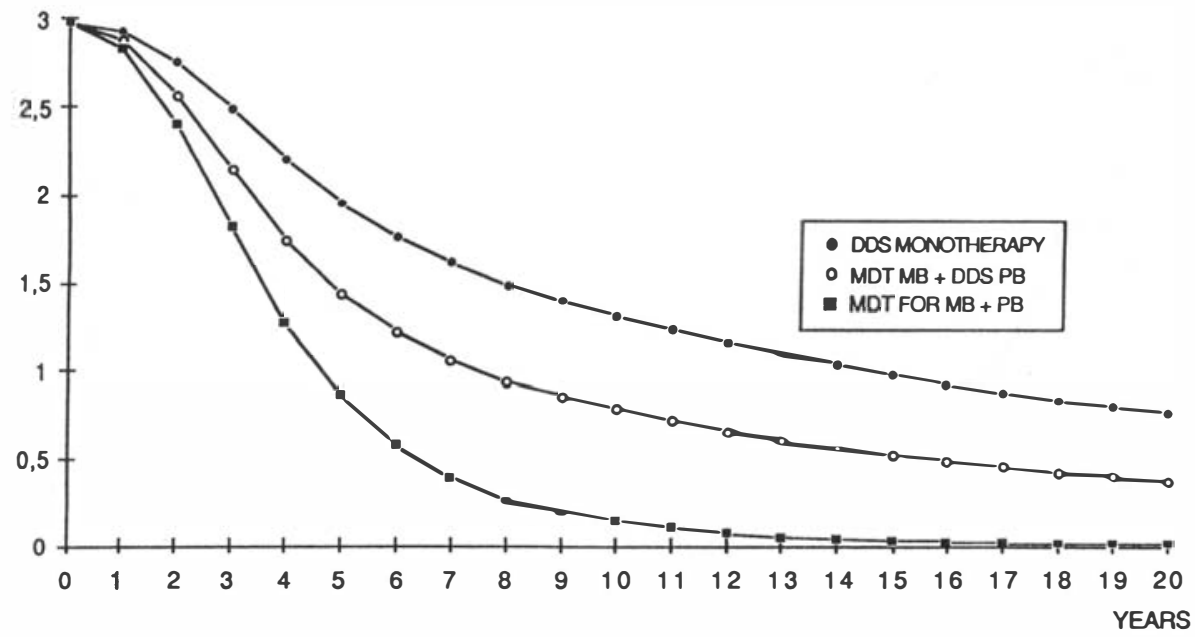

Figure 6. Computer simulation of incidence of leprosy with three different therapeutic regimens. Reproduced by kind permission of the International Journal of Leprosy. ${ }^{4}$

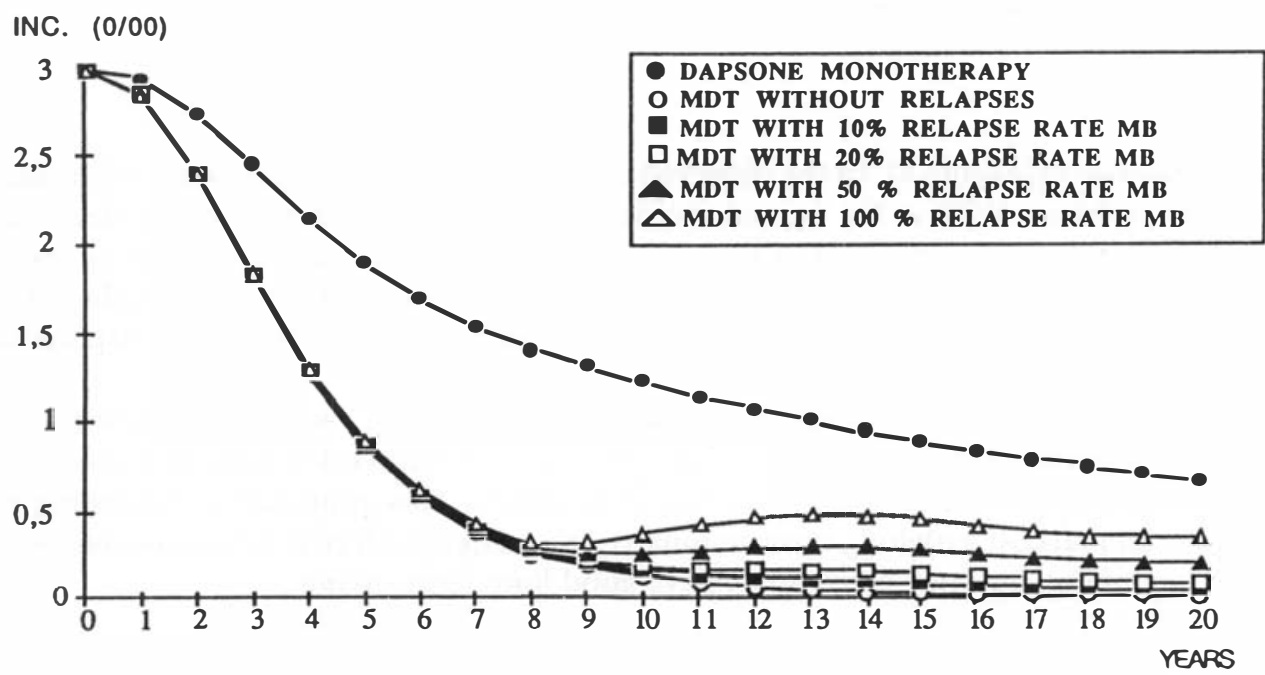

Figure 7. Computer simulation of incidence with dapsone and MDT with different relapse rates.

reversed for a period (with a $50-100 \%$ relapse rate) only after 8 years. These results are important in view of a popular claim that multibacillary patients under MDT should continue treatment after 2 years even when they become bacillary negative. Some say they should even be treated for life. As for control, relapses are not significant as long as they are detected early; in other terms, all treatment requires a close surveillance system.

Simulation of resistance required the introduction of new compartments in the model and an extension of 50 years (which probably has little justification due to the long-term 


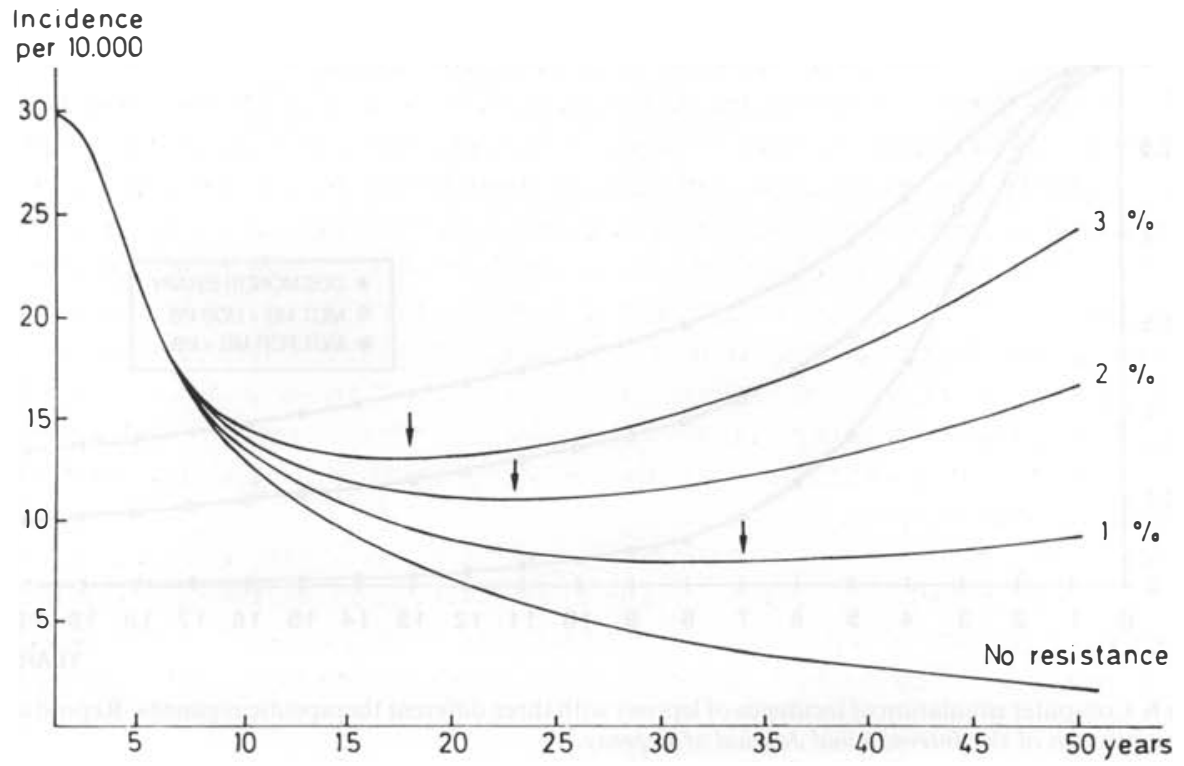

Figure 8. Prediction of incidence for both types of leprosy with $1-2-3 \%$ secondary drug resistance. Reproduced by kind permission of the International Journal of Leprosy. ${ }^{3}$

unpredictability) (Figure 8). In the observed conditions, secondary resistance constitutes a major problem. With a $3 \%$ annual incidence of secondary resistance, the declining trends in incidence slow down after 6 or 7 years and then begin rising at increasing speed after 15 years. These results are more forboding because no provision was made in the very simplified model for the contribution of subsequent cases with primary resistance to incidence in subsequent years.

Cost-benefit analysis can also be simulated by introducing various cost-parameters. Such econometric analysis should be subject to caution, for it is often difficult to calculate the various components of different control strategies. This approach is interesting to compare annual cost with long-term cumulative cost. Highly effective strategies are costly at the beginning, but may prove to achieve good long-term saving.

\section{Conclusion}

Overall, we must consider the possible uses and drawbacks of the model. It was developed in a specific area, South India, which presents its own special epidemiological contexts. Therefore it has clear limitations.

The resulting figures should be viewed with caution. The results are highly sensitive to a number of parameters, either directly observed or derived through statistical estimation. The model is based on a number of assumptions, not all verifiable and possibly too simplified. The quantitative results should not be generalized for use in other areas which might have different conditions of prevalence, incidence, multi/paucibacillary ratios, 
birth and death rates. It is not certain that epidemiological data with an equal value to those collected in South India could be found in many places.

The model serves its own purpose and might not be best used when repeating the exercise exactly in different places. It acts as a type of grammar or structure. The model helps to clarify epidemiological concepts as exemplified by the existing differences between immunoprophylactic and immunotherapeutic vaccines or the epidemiological similarity of noncompliance and treatment-sensitive relapse albeit on different time scales. The model's most important purpose is to list the problems it tackles in the order of importance and make various comparisons. It could consider whether every patient should receive MDT, or only multibacillary patients; it could compare the relative insensitivity of incidence to early detection (despite the complete and early detection in the study area); or it could stress the importance of resistance in the long term as compared to nondrug resistant relapses.

Due to the present success of MDT-based control, this modelling approach in leprosy is no longer a part of basic research. The future belongs to microscale modelling for disappearing diseases. What can be expected in terms of limited foci, clusters, and erratic time fluctuations? This could be called, to use a fashionable new avenue of research, fractal epidemiology.

\section{References}

1 Doull JA et al. The Incidence of Leprosy in Cordova and Talisay, Cebu, PI, Int J Lepr, 1942; 10: $107-31$.

${ }^{2}$ Lechat MF et al. Un modèle épidémiométrique de la lèpre Bull 'Organisation mondiale Santé, 1974; 51: 361-73.

3 Lechat MF et al. Simulation of vaccination and resistance in leprosy using an epidemiometric model, Int $J$ Lepr, 1985; 53: 461-7.

4 Lechat MF et al. Selection of MDT strategies through epidemiometric modeling, Int J Lepr, 1990; 58; 296301. 